\title{
Diabetes mellitus auto-referido no Município de São Paulo: prevalência e desigualdade
}

\author{
Self-reported diabetes mellitus in the city of \\ São Paulo: prevalence and inequality
}

Paul ete Gol denberg 1

Laércio J. Franco 1

Heloisa Pagliaro 1

Rebeca de S. Silva 1

Carla A. dos Santos 1

\footnotetext{
1 Departamento de Medicina Preventiva, Escola Paulista de Medicina, Universidade Federal de São Paulo. Rua Pedro Toledo 675, São Paulo, SP 04023-062, Brasil.
}

Resumo Constitui objetivo do presente trabal ho a caracterização da prevalência do Diabetes Mellitus Auto-Referi do (DMAR) no Município de São Paulo, obti da a partir do inquérito domiciliar realizado pel o Estudo Multi cêntrico sobre a Prevalência do Diabetes no Brasil , de 1986 a 1988. A amostra foi composta por 2007 indivíduos de 30 a 69 anos de idade, de ambos os sexos, selecionados em três áreas de disti ntas condi ções sociais, nas quais estavam sendo implantados programas assi stenciais aos diabéticos. A prevalência, obtida através de gl icemia capilar, $2 \mathrm{~h}$ após sobrecarga de $75 \mathrm{~g}$ de glicose, foi de 9,7\%. A prevalência do DMAR foi de 4,7\%, tendo sido observado aumento de acordo com a idade, e estreita rel ação com o relato de história familiar de diabetes. Observou-se, ainda, diferença significante segundo o sexo (3,5\% na população masculina e 5,7\% na feminina), concentrando-se mai ores valores nos níveis sócio-econômicos mais elevados, na população masculina, e, nos níveis mais baixos, na população feminina.

Palavras-chave Diabetes Mellitus; Epidemiologia; Inquérito domiciliar

Abstract This report analyzes characteristics of self-reported diabetes mellitus in the city of São Paulo, Brazil. The data were obtained from the Brazilian Multicenter Study on Prevalence of Diabetes Mellitus, a hou sehold survey performed in 1986-88. The São Paulo sample consisted of 2,007 individuals aged 30-69 years, of both sexes, sel ected from three areas with distinct socioeconomic level s. The estimated preval ence using a $75 \mathrm{~g} g l$ ucose load and measurement of twohour capillary glycemia was $9.7 \%$. Preval ence of self-reported diabetes was $4.7 \%$ and increased with age and presence of family history of diabetes. There was a significant difference between sexes (3.5\% in men and $5.7 \%$ in women), with higher rates of self-reported diabetes at higher economic levels among men and higher rates at lower socio-economic levels among women.

Key words Diabetes Mellitus; Epidemiology; Household Survey 


\section{Introdução}

O Estudo Multicêntrico sobre a Prevalência do Diabetes no Brasil -EM PDB (Malerbi \& Franco, 1992), realizado em nove capitais, no período de 1986 a 1988, na população de 30 a 69 anos de idade, evidenciou a prevalência de $7,6 \%$ para o conjunto da amostra, sendo de $9,7 \%$ a prevalência estimada para São Paulo, que apresentou o mai or valor dentre as áreas estudadas.

Para o conjunto da amostra do EM PDB, não se observaram diferenças na prevalência quanto ao sexo e à escolaridade quando os coeficientes foram ajustados por idade. Recolocase esta questão na caracterização da prevalência da doença em São Paulo, tendo em vista as especificidades regionais de sua manifestação. Assim, constitui objetivo do presente trabalho a caracterização da prevalência do diabetes mellitus em São Paulo, do ponto de vista das distinções sócio-econômicas e de gênero.

Considerando, por sua vez, um dos propósitos do estudo o de promover interven ção precoce e melhorar o controle do diabetes em nosso meio, será destacada a doença auto-referida ou pré-diagnosticada, tendo em vista seu dimensionamento entre os usuários dos serviços de saúde, na forma como estão estruturados, para o diagnóstico do diabetes.

\section{Procedimentos de investigação}

O EMPDB constou da realização de inquérito domiciliar, envolvendo, numa primeira fase, um rastreamento mediante glicemia capilar de jejum (GCJ) e, numa segunda fase, a determinação de glicemia capilar $2 \mathrm{~h}$ após sobrecarga de $75 \mathrm{~g}$ de glicose em indivíduos com $\mathrm{GCJ} \geq 100 \mathrm{mg} / \mathrm{dl}$ e em $1 / 6$ dos indivíduos com $\mathrm{GCJ}<100 \mathrm{mg} / \mathrm{dl}$. Descrição detalhada dos procedimentos e do processo de sel eção amostral adotados nesta investigação foi publicada por Malerbi \& Franco (1992).

Para efeito da composição da amostra do distrito de São Paulo foram escolhidas três áreas: Vila Mariana, Saúde e Santo Amaro, atendendo à estrutura social diferenciada de acordo com a constituição de áreas homogêneas (Bussab \& Dini, 1985) e nas quais estavam sendo implantados programas de assistência aos diabéticos, nas unidades da Secretaria de Estado da Saúde. Esta disposição tinha em vista não só a realização de futuras avaliações, mas, sobretudo, a necessidade ética de fornecimento de retaguarda assistencial ao diagnóstico da doença na população estudada. Obedecen- do à combinação de critérios de limitação orçamentária e precisão das estimativas, foram sorteados $20 \%$ dos setores censitários de cada área, nos quais se realizou um levantamento dos domicílios residenciais. Para se obter o número de indivíduos definido no plano amostral, com base em informações do IBGE sobre o número médio de indivíduos na faixa etária de 30 a 69 anos por setor censitário, foram sorteados $20 \%$ dos domi cílios enumerados na etapa anterior.

Foram sorteados 2020 domicílios. Destes, em 590 (29,2\%) não residiam indivíduos na faixa de 30 a 69 anos de idade; em 119 (5,9\%), os residentes se recusaram a participar do estudo; e 156 domicílios (7,7\%) estavam desocupados. Nos 1155 domicílios restantes foram enumeradas 6771 pessoas.

Das 6771 pessoas enumeradas, 2577 (38,1\%) foram consideradas elegíveis, ou seja, integravam população de ambos os sexos, na faixa de 30 a 69 anos de idade. As gestantes foram excluídas, devido às exigências diferenciadas de diagnóstico. Dentre os elegíveis, 385 (14,9\%) recusaram-se a participar do inquérito e 185 $(7,2 \%)$ constituíram-se em perdas, restando, assim, os 2007 indivíduos que fizeram parte da fase de rastreamento. Entre estes, $877(43,8 \%)$ eram homens e 1129 (56,2\%) mulheres.

Durante a fase de rastreamento, paralelamente à realização da glicemia de jejum, foi possível identificar os casos de diabetes já diagnosticados. Na ocasião, foram levantadas informações a respeito da idade, cor, escolaridade, ocupação, condições mórbidas ou incapacitantes, uso de medicamentos e existência de familiares com diabetes, especificando-se o grau de parentesco. A cor foi identificada pelo entrevistador, sendo posteriormente agrupada em brancos, negros (pretos, mulatos, pardos e cafusos) e outros (orientais).

Com base nestes dados, estimou-se a prevalência do diabetes mellitus auto-referido (DMAR), ressaltando-se, nestes casos, a exigência da confirmação diagnóstica, mediante verificação de resultados de glicemias prévias. A caracterização sócio-econômica da prevalência do DM AR envolveu a consideração, a par da idade e do sexo, da escolaridade, da naturalidade, da ocupação, da cor, bem como da distribuição por área. A inclusão da história familiar de diabetes (presença ou não) na análise dos dados decorreu da importância a ela atribuída por vários estudos (WHO, 1985; Everhart et al., 1985 e Harris, 1987), bem como pelo próprio EM PDB, diante do caráter interveniente que desempenharia na epidemiologia do diabetes. 
Para efeito de comparação da caracterização sócio-econômica, levaram-se em consideração as estatísticas publicadas pela Fundação IBGE sobre a Região Metropolitana de São PauIo, nos anos 80 (FIBGE, 1983, 1988, 1990). Os coeficientes de prevalência foram ajustados por idade, pelo método direto, usando como padrão a população do país em 1980.

$\mathrm{Na}$ análise estatística, priorizando a consideração de cada variável para caracterização da amostra e sua relação com o DMAR, utilizou-se o teste de $\chi^{2}$ e Fisher, apresentando-se o nível de significância calculado.

Através do método preconizado por Higgins \& Koch (1975), ordenaram-se as variáveis relacionadas com o DMAR em função dos maiores valores do $\chi^{2}$, considerando as variáveis dicotomizadas. Selecionada a primeira variável, foram observadas as variações residuais das variáveis subseqüentes. Repetindo-se este processo sucessivamente, estabeleceram-se as variáveis a serem incluídas no modelo, por ordem de importância.

Estabelecida a hierarquização das variáveis, calculou-se o odds-ratio de cada combinação, em relação à casela inicial de referência.

\section{Resultados}

\section{Características sócio-demográficas}

De acordo com os dados especificados na Tabela 1, observou-se semel hança de composição por idade e sexo entre a população amostral e a da Região Metropolitana de São Paulo (RMSP) em 1987/88.

Quanto ao sexo, as populações da amostra e da RMSP assemelharam-se no sentido de apresentarem uma superioridade numérica de muIheres (56,3\% na amostra e 53,0\% na RMSP). As razões de sexo da população selecionada apontaram para a predominância de mulheres em todos os grupos etários. Esta tendência também se verificou na população da RMSP. As razões de sexo da amostra foram inferiores às da RMSP em todos os grupos etários, com exceção da faixa de 50-59 anos.

Com relação aos níveis de instrução, os analfabetos estiveram presentes em $12,0 \%$ da amostra, e 17,6\% haviam cursado entre um a três anos de estudos, totalizando $29,6 \%$ na faixa de menores níveis de instrução; 30,2\% dos integrantes da amostra concentraram-se na

Tabela 1

Características sócio-demográficas da população selecionada. EMPDMB/Fase 1 - Amostra São Paulo, 1986/87, e população da região metropolitana de São Paulo (RMSP), 1987/88.

\begin{tabular}{|c|c|c|c|c|c|c|c|}
\hline \multirow[t]{2}{*}{ Variáveis } & & \multicolumn{3}{|c|}{ Amostra } & \multicolumn{3}{|c|}{ RM SP } \\
\hline & & $\begin{array}{c}\text { Total } \\
(\mathrm{n}=2007) \\
(\%)\end{array}$ & $\begin{array}{c}\text { Homens } \\
(n=878) \\
(\%)\end{array}$ & $\begin{array}{c}\text { Mulheres } \\
(n=1129) \\
(\%)\end{array}$ & $\begin{array}{c}\text { Total } \\
(\mathrm{n}=5911157) \\
(\%)\end{array}$ & $\begin{array}{c}\text { Homens } \\
(\mathrm{n}=2776238) \\
(\%)\end{array}$ & $\begin{array}{c}\text { Mulheres } \\
(\mathrm{n}=3134919) \\
(\%)\end{array}$ \\
\hline Idade & $\begin{array}{l}30-39 \\
40-49 \\
50-59 \\
60-69\end{array}$ & $\begin{array}{l}38,6 \\
28,0 \\
21,5 \\
12,0\end{array}$ & $\begin{array}{l}39,5 \\
25,4 \\
23,9 \\
11,2\end{array}$ & $\begin{array}{l}37,8 \\
30,0 \\
19,6 \\
12,6\end{array}$ & $\begin{array}{l}38,5 \\
28,9 \\
21,4 \\
11,3\end{array}$ & $\begin{array}{l}38,1 \\
29,1 \\
21,4 \\
11,4\end{array}$ & $\begin{array}{l}38,8 \\
28,8 \\
21,3 \\
11,1\end{array}$ \\
\hline O cupação & $\begin{array}{l}\text { O cupados } \\
\text { Não-O cupados } \\
\text { Donas-de-Casa } \\
\text { Aposentados }\end{array}$ & $\begin{array}{l}63,4 \\
36,6 \\
82,4 \\
18,3\end{array}$ & $\begin{array}{r}88,1 \\
11,9 \\
0,0 \\
100,0\end{array}$ & $\begin{array}{r}44,7 \\
55,3 \\
95,0 \\
5,0\end{array}$ & $\begin{array}{l}60,0 \\
40,0 \\
- \\
-\end{array}$ & $\begin{array}{l}84,5 \\
15,5 \\
- \\
-\end{array}$ & $\begin{array}{l}38,5 \\
61,5 \\
- \\
-\end{array}$ \\
\hline Anos de Estudo & $\begin{array}{l}\text { S/ Instr. } \\
1-3 \\
4-7 \\
8-10 \\
11 \geq\end{array}$ & $\begin{array}{l}12,0 \\
17,7 \\
30,2 \\
11,3 \\
28,9\end{array}$ & $\begin{array}{l}11,3 \\
14,3 \\
30,1 \\
12,2 \\
32,2\end{array}$ & $\begin{array}{l}12,6 \\
20,3 \\
30,2 \\
10,5 \\
26,4\end{array}$ & $\begin{array}{l}10,0 \\
25,7 \\
32,3 \\
11,8 \\
20,2\end{array}$ & $\begin{array}{r}8,1 \\
24,4 \\
32,0 \\
11,6 \\
23,9\end{array}$ & $\begin{array}{l}11,6 \\
26,9 \\
32,6 \\
12,0 \\
16,9\end{array}$ \\
\hline Naturalidade & $\begin{array}{l}\text { São Paulo } \\
\text { Fora São Paulo }\end{array}$ & $\begin{array}{l}25,5 \\
74,5\end{array}$ & $\begin{array}{l}26,8 \\
73,2\end{array}$ & $\begin{array}{l}24,5 \\
75,6\end{array}$ & $\begin{array}{l}- \\
-\end{array}$ & - & - \\
\hline Cor & $\begin{array}{l}\text { Brancos } \\
\text { Negros } \\
\text { Outros } \\
\text { Ignorada }\end{array}$ & $\begin{array}{r}73,5 \\
22,8 \\
3,4 \\
0,3\end{array}$ & $\begin{array}{r}74,2 \\
22,6 \\
3,1 \\
0,2\end{array}$ & $\begin{array}{r}73,1 \\
23,0 \\
3,6 \\
0,3\end{array}$ & $\begin{array}{r}74,1 \\
23,2 \\
2,8 \\
-\end{array}$ & $\begin{array}{r}72,9 \\
24,1 \\
2,9 \\
-\end{array}$ & $\begin{array}{c}75,1 \\
22,3 \\
2,6 \\
-\end{array}$ \\
\hline
\end{tabular}

Fonte: EPM/MS - Estudo Multicêntrico sobre a prevalência de diabetes mellitus no Brasil

FIBGE - Pesquisa de O rçamentos Familiares - POF - 1987/88. FIBGE - Pesquisa Nacional por Amostra de Domicílios - PNAD - 1987/88. 
Tabela 2

Características sócio-demográficas da população selecionada, segundo áreas. EMPDMB/Fase 1 - Amostra São Paulo - $1986 / 87$.

\begin{tabular}{|c|c|c|c|c|c|c|c|c|c|c|}
\hline \multirow[t]{2}{*}{ Variáveis } & & \multicolumn{3}{|c|}{ Vila Mariana } & \multicolumn{3}{|c|}{ Saúde } & \multicolumn{3}{|c|}{ Santo Amaro } \\
\hline & & $\begin{array}{c}\mathrm{n}=284 \\
\mathrm{M} \text { asc. } \\
(\%)\end{array}$ & $\begin{array}{c}n=379 \\
\text { Fem. } \\
(\%)\end{array}$ & $\begin{array}{c}\mathrm{n}=663 \\
\text { Total } \\
(\%)\end{array}$ & $\begin{array}{c}n=235 \\
\text { Masc. } \\
(\%)\end{array}$ & $\begin{array}{c}\mathrm{n}=311 \\
\text { Fem. } \\
(\%)\end{array}$ & $\begin{array}{c}n=546 \\
\text { Total } \\
(\%)\end{array}$ & $\begin{array}{c}n=359 \\
M \text { asc. } \\
(\%)\end{array}$ & $\begin{array}{c}n=439 \\
\text { Fem. } \\
(\%)\end{array}$ & $\begin{array}{c}n=798 \\
\text { Total } \\
(\%)\end{array}$ \\
\hline Sexo & & 42,8 & 57,2 & 100,0 & 43,0 & 57,0 & 100,0 & 45,0 & 55,0 & 100,0 \\
\hline Idade & $\begin{array}{l}30-39 \\
40-49 \\
50-59 \\
60-69\end{array}$ & $\begin{array}{l}35,6 \\
28,2 \\
21,1 \\
15,1\end{array}$ & $\begin{array}{l}36,7 \\
26,7 \\
19,5 \\
17,2\end{array}$ & $\begin{array}{l}36,2 \\
27,3 \\
20,2 \\
16,3\end{array}$ & $\begin{array}{l}48,5 \\
20,9 \\
18,3 \\
12,3\end{array}$ & $\begin{array}{l}37,6 \\
28,0 \\
22,2 \\
12,2\end{array}$ & $\begin{array}{l}42,3 \\
24,9 \\
20,5 \\
12,3\end{array}$ & $\begin{array}{r}36,8 \\
26,2 \\
29,8 \\
7,2\end{array}$ & $\begin{array}{r}39,0 \\
34,4 \\
17,8 \\
8,9\end{array}$ & $\begin{array}{r}38,0 \\
30,7 \\
23,2 \\
8,2\end{array}$ \\
\hline Anos de Estudo & $\begin{array}{l}\text { S/Instrução } \\
1-3 \\
4-7 \\
8-10 \\
11 \geq \\
\text { S/Informação }\end{array}$ & $\begin{array}{r}1,4 \\
1,1 \\
12,3 \\
13,4 \\
71,8 \\
0,0\end{array}$ & $\begin{array}{r}1,6 \\
5,5 \\
18,2 \\
14,0 \\
60,7 \\
0,0\end{array}$ & $\begin{array}{r}1,5 \\
3,6 \\
15,7 \\
13,7 \\
65,5 \\
0,0\end{array}$ & $\begin{array}{r}10,6 \\
17,5 \\
43,4 \\
12,8 \\
15,3 \\
0,4\end{array}$ & $\begin{array}{r}15,4 \\
27,7 \\
37,6 \\
9,0 \\
10,3 \\
0,0\end{array}$ & $\begin{array}{r}13,4 \\
23,3 \\
40,1 \\
10,6 \\
12,5 \\
0,2\end{array}$ & $\begin{array}{r}19,5 \\
22,6 \\
35,4 \\
10,9 \\
11,7 \\
0,0\end{array}$ & $\begin{array}{r}20,1 \\
27,8 \\
35,3 \\
8,7 \\
8,2 \\
0,0\end{array}$ & $\begin{array}{r}19,8 \\
25,4 \\
35,3 \\
9,7 \\
9,8 \\
0,0\end{array}$ \\
\hline Naturalidade & $\begin{array}{l}\text { São Paulo } \\
\text { Fora São Paulo }\end{array}$ & $\begin{array}{l}42,6 \\
57,4\end{array}$ & $\begin{array}{l}40,9 \\
59,1\end{array}$ & $\begin{array}{l}41,6 \\
58,4\end{array}$ & $\begin{array}{l}23,4 \\
76,6\end{array}$ & $\begin{array}{l}18,7 \\
81,4\end{array}$ & $\begin{array}{l}20,7 \\
79,3\end{array}$ & $\begin{array}{l}16,4 \\
83,6\end{array}$ & $\begin{array}{l}14,4 \\
85,7\end{array}$ & $\begin{array}{l}15,3 \\
84,7\end{array}$ \\
\hline Cor & $\begin{array}{l}\text { Brancos } \\
\text { Negros } \\
\text { Outros } \\
\text { S/Informação }\end{array}$ & $\begin{array}{r}89,1 \\
4,6 \\
6,0 \\
0,4\end{array}$ & $\begin{array}{r}87,6 \\
5,5 \\
6,3 \\
0,5\end{array}$ & $\begin{array}{r}88,2 \\
5,1 \\
6,2 \\
0,5\end{array}$ & $\begin{array}{r}79,2 \\
18,7 \\
1,7 \\
0,4\end{array}$ & $\begin{array}{r}79,1 \\
19,0 \\
1,6 \\
0,3\end{array}$ & $\begin{array}{r}79,1 \\
18,9 \\
1,7 \\
0,4\end{array}$ & $\begin{array}{r}59,1 \\
39,3 \\
1,7 \\
0,0\end{array}$ & $\begin{array}{r}56,3 \\
41,0 \\
2,7 \\
0,0\end{array}$ & $\begin{array}{r}57,5 \\
40,2 \\
2,3 \\
0,0\end{array}$ \\
\hline O cupação & $\begin{array}{l}\text { O cupados } \\
\text { Não-O cupados }\end{array}$ & $\begin{array}{l}88,0 \\
12,0\end{array}$ & $\begin{array}{l}45,7 \\
54,4\end{array}$ & $\begin{array}{l}63,8 \\
36,2\end{array}$ & $\begin{array}{l}84,3 \\
15,7\end{array}$ & $\begin{array}{l}35,1 \\
65,0\end{array}$ & $\begin{array}{l}56,2 \\
43,8\end{array}$ & $\begin{array}{r}90,8 \\
9,2\end{array}$ & $\begin{array}{l}50,6 \\
49,4\end{array}$ & $\begin{array}{l}68,7 \\
31,3\end{array}$ \\
\hline
\end{tabular}

Fonte: Estudo Multicêntrico sobre a prevalência do diabetes mellitus no Brasil.

faixa de quatro a sete anos de estudo; e 28,9\%, na faixa de 11 anos e mais. Os homens apresentaram maiores níveis de instrução do que as mulheres, ressaltando-se que o padrão educacional da amostra também coincidiu com o da população da RMSP.

Quanto ao local de nascimento, 25,5\% eram naturais do Município de São Paulo, sendo os restantes naturais de outros municípios do Estado de São Paulo, de outros estados ou de outros países. Comparações com os dados da RMSP foram inviabilizadas devido à inexistência de informações relativas a essa variável. Vale dizer que, entre os naturais do Município de São Paulo, concentraram-se níveis mais altos de escolaridade: $72,3 \%$ da população masculina e $61,2 \%$ da população feminina apresentavam escolaridade acima de oito anos de estudo, ao passo que estas proporções entre os migrantes foram, respectivamente, 34,1\% e $29,1 \%$, sendo as diferenças estatisticamente significantes ( $p \varangle 0,001)$.

Entre os indivíduos selecionados, 73,5\% eram brancos, $22,8 \%$, negros, sendo os restantes incluídos na categoria "outros". A amostra também refletiu o padrão da composição por cor da população da RMSP, tanto para a população masculina como para a feminina. Ressal- ta-se também a associação da cor com a escolaridade, dando conta das distinções sócioeconômicas relacionadas à consideração desta variável. Enquanto entre os brancos as proporções de indivíduos nos níveis mais baixos e mais altos de escolaridade foram semelhantes, entre os negros, $85 \%$ apresentavam escolaridade inferior a oito anos de estudo. Esta tendência se repetiu tanto na população masculina, como na feminina, observando-se sempre valores menores de escolaridade entre as mulheres.

Quanto à ocupação, os resultados mostraram que $63,4 \%$ dos indivíduos exerciam atividades remuneradas. Entre os homens, 88,1\% trabalhavam, sendo os demais aposentados. Entre as mulheres, 44,7\% estavam inseridas no mercado de trabalho, sendo que, entre as nãoocupadas, 95\% eram donas-de-casa, e apenas $5 \%$ incluíam-se na categoria de aposentadas. I nformações sobre a RMSP indicam que o nível de ocupação foi ligeiramente maior na amostra estudada.

Embora a escolha das áreas não tivesse obedecido a um critério aleatório, estes dados apontaram para a semelhança entre o perfil do conjunto da amostra e da população da RMSP, principalmente no que tange ao padrão etário, ao sexo, à instrução e à cor. 
São significativas, por sua vez, as combinações destas variáveis, observadas no espaço socialmente delimitado das áreas, configurando estratos constitutivos do conjunto amostral. De acordo com os dados da Tabela 2, observou-se que Vila Mariana apresentou a maior proporção de indivíduos com mais de sessenta anos e esta proporção diminuiu progressivamente na Saúde e em Santo Amaro. Observouse a mesma tendência quanto à proporção de integrantes da amostra com maior escolaridade e, inversamente, com a proporção dos sem instrução ou com menos de três anos de estudo (primário incompleto). A proporção de naturais de São Paulo e de brancos, que apresentavam maiores níveis de escolaridade, acompanhou, coerentemente, este mesmo comportamento, o que permite caracterizar os níveis decrescentes de condições de existência, no sentido seqüencial da Vila Mariana, Saúde e Santo Amaro, ratificando os critérios diferenciais de condições sócio-econômicas adotados na escolha das áreas.

O comportamento observado com relação às referidas variáveis entre as áreas aplica-se tanto para a população masculina, quanto para a femilina, ressaltando-se que as diferenças observadas foram estatisticamente significantes $(p<0,01)$.

No caso da não-ocupação, não se observou linearidade, seja no sentido de aumento ou de diminuiç̧ão das proporções entre as áreas. Os maiores valores concentraram-se na área de condição sócio-econômica intermediária, a da Saúde, tanto na população do sexo masculino como na do feminino. As diferenças encontradas, porém, só foram estatisticamente significantes na população feminina $(p \varangle 0,01)$.

Prevalência de diabetes mellitus auto-referido

A Prevalência do DMAR na amostra foi de 4,7\%, sendo de 3,5\% na população masculina e de $5,7 \%$ na feminina (Tabela 3 ). Estes valores representaram, respectivamente, $50,5 \%$ da prevalência estimada para o conjunto populacional; $40,2 \%$ da estimada para a população masculina; e $58,8 \%$ da estimada para a feminina. Ressaltam-se, nestas circunstâncias, maiores proporções de desconhecimento da presença da doença entre os homens.

Não houve mudanças substanciais nas prevalências do DMAR com o ajuste dos coeficientes por idade $(4,7 \%$ para o conjunto populacional, 3,3\% para a população masculina e 5,8\% para a população feminina). Destaca-se o aumento progressivo da prevalência do DMAR com o avanço da idade ( $p<0,001$ ) e sua ocorrência mais elevada na população feminina $(p \triangleleft 0,05)$.

Quanto à situação ocupacional, os nãoocupados apresentaram, sistematicamente, prevalência mais elevada que os ocupados ( $p<$ $0,001)$, seja na população masculina ( $<\varangle 0,001)$, seja na feminina ( $p<0,01)$, mesmo quando os coeficientes foram ajustados por idade.

Merece ser destacado que, na categoria de não-ocupados, configurou-se a única situação em que os homens, mesmo no caso dos dados ajustados, apresentaram uma prevalência mais elevada do que as mulheres ( $10,7 \%$ e $6,4 \%$, respectivamente), sendo esta diferença estatisticamente significante $(p \measuredangle 0,05)$.

Levando em consideração a escolaridade, observamos que o DMAR se distribuiu de forma irregular nas diversas categorias, não tendo sido observadas diferenças estatisticamente significantes para o conjunto amostral. Na população masculina, os valores da prevalência de DMAR nos diferentes níveis de escolaridade estiveram, invariavelmente, em torno de $3 \%$. Na população feminina, por seu lado, as maiores freqüências concentraram-se nos níveis mais baixos de escolaridade. As diferenças observadas não foram estatisticamente significantes quando os dados foram analisados segundo as categorias adotadas; porém, o mesmo não aconteceu quando estas categorias foram agregadas abaixo e acima de oito anos de estudo. Nestas circunstâncias, as diferenças foram estatisticamente significantes $(p<0,05)$ para o conjunto da amostra e para a população feminina.

Os naturais do município de São Paulo apresentaram prevalência de DMAR semelhante à dos migrantes $(4,75 \%$ e $4,6 \%$, respectivamente). Comparando-se as diferenças de gênero, em que pese não terem significância estatística, observou-se, na população masculina, maior prevalência do DMAR entre os naturais do Município de São Paulo e esta diferença se acentuou após o ajuste dos coeficientes por idade; entre as mulheres, aconteceu o inverso: os maiores valores concentraram-se entre as migrantes.

Levando-se em consideração a cor, observou-se a presença de valores relativamente maiores de prevalência de DMAR na população branca $(4,3 \%)$ do que na negra (3,8\%). Este padrão se repetiu na população masculina mas não na feminina, sendo que as diferenças não foram estatisticamente significantes.

Considerando a prevalência do DMAR segundo áreas que apresentavam configurações diferenciadas quanto às variáveis anteriormen- 
Tabela 3

Prevalência de diabetes mellitus auto-referido em homens e mulheres, segundo Idade, situação ocupacional, anos de estudos, naturalidade, cor, área e história familiar de diabetes. EMPDMB - Fase 1 - Amostra São Paulo, 1986/87.

\begin{tabular}{|c|c|c|c|c|c|c|c|}
\hline \multirow[t]{2}{*}{ Variáveis } & & \multicolumn{3}{|c|}{ Prevalência Bruta (\%) } & \multicolumn{3}{|c|}{ Prevalência Ajustada (\%) } \\
\hline & & $\begin{array}{c}\text { Total } \\
(n=2007)\end{array}$ & $\begin{array}{l}\text { Homens } \\
(n=878)\end{array}$ & $\begin{array}{l}\text { Mulheres } \\
(n=1129)\end{array}$ & Total & Homens & Mulheres \\
\hline Auto-Referido & & 4,7 & 3,5 & 5,7 & 4,7 & 3,3 & 5,8 \\
\hline Total Estimado & & 9,3 & 8,7 & 9,7 & 9,0 & 8,4 & 9,9 \\
\hline Idade & $\begin{array}{l}30-39 \\
40-49 \\
50-59 \\
60-69\end{array}$ & $\begin{array}{r}1,8 \\
2,9 \\
9,5 \\
10,0\end{array}$ & $\begin{array}{l}0,3 \\
2,7 \\
7,6 \\
8,2\end{array}$ & $\begin{array}{r}3,0 \\
3,0 \\
11,3 \\
11,3\end{array}$ & $\begin{array}{l}- \\
- \\
- \\
-\end{array}$ & $\begin{array}{l}- \\
- \\
- \\
-\end{array}$ & $\begin{array}{l}- \\
- \\
- \\
-\end{array}$ \\
\hline O cupação & $\begin{array}{l}\text { O cupados } \\
\mathrm{N} \text { ão-O cupados }\end{array}$ & $\begin{array}{l}2,7 \\
8,2\end{array}$ & $\begin{array}{r}2,2 \\
13,5\end{array}$ & $\begin{array}{l}3,6 \\
7,4\end{array}$ & $\begin{array}{l}3,1 \\
6,7\end{array}$ & $\begin{array}{r}2,4 \\
10,7\end{array}$ & $\begin{array}{l}4,4 \\
6,4\end{array}$ \\
\hline Anos de Estudo & $\begin{array}{l}\text { S/Instrução } \\
1-3 \\
4-7 \\
8-10 \\
11 \geq\end{array}$ & $\begin{array}{l}5,0 \\
6,8 \\
5,1 \\
3,1 \\
3,6\end{array}$ & $\begin{array}{l}3,0 \\
3,2 \\
3,8 \\
3,7 \\
3,6\end{array}$ & $\begin{array}{l}6,3 \\
8,7 \\
6,2 \\
2,5 \\
3,7\end{array}$ & $\begin{array}{l}4,4 \\
6,5 \\
5,2 \\
3,3 \\
4,7\end{array}$ & $\begin{array}{l}1,2 \\
3,0 \\
3,5 \\
3,5 \\
4,6\end{array}$ & $\begin{array}{l}6,9 \\
8,4 \\
6,7 \\
2,6 \\
4,7\end{array}$ \\
\hline N aturalidade & $\begin{array}{l}\text { São Paulo } \\
\text { Fora São Paulo }\end{array}$ & $\begin{array}{l}4,3 \\
4,9\end{array}$ & $\begin{array}{l}4,3 \\
3,3\end{array}$ & $\begin{array}{l}4,4 \\
6,1\end{array}$ & $\begin{array}{l}4,8 \\
4,6\end{array}$ & $\begin{array}{l}5,0 \\
2,8\end{array}$ & $\begin{array}{l}4,4 \\
6,1\end{array}$ \\
\hline Cor & $\begin{array}{l}\text { Brancos } \\
\text { Negros } \\
\text { Outros }\end{array}$ & $\begin{array}{l}5,2 \\
3,7 \\
2,9\end{array}$ & $\begin{array}{l}4,2 \\
2,0 \\
0,0\end{array}$ & $\begin{array}{l}5,9 \\
5,0 \\
4,9\end{array}$ & $\begin{array}{l}4,3 \\
3,8 \\
3,2\end{array}$ & $\begin{array}{l}4,0 \\
1,8 \\
0,0\end{array}$ & $\begin{array}{l}4,6 \\
5,4 \\
6,2\end{array}$ \\
\hline Áreas & $\begin{array}{l}\text { V. Mariana } \\
\text { Saúde } \\
\text { Sto. Amaro }\end{array}$ & $\begin{array}{l}5,1 \\
5,7 \\
3,8\end{array}$ & $\begin{array}{l}6,7 \\
1,7 \\
2,2\end{array}$ & $\begin{array}{l}4,0 \\
8,7 \\
5,0\end{array}$ & $\begin{array}{l}5,2 \\
5,8 \\
4,0\end{array}$ & $\begin{array}{l}7,2 \\
1,8 \\
1,9\end{array}$ & $\begin{array}{l}3,8 \\
8,5 \\
5,6\end{array}$ \\
\hline História Familiar & $\begin{array}{l}\text { Sim } \\
\text { Não }\end{array}$ & $\begin{array}{l}8,5 \\
2,8\end{array}$ & $\begin{array}{l}7,1 \\
2,1\end{array}$ & $\begin{array}{l}9,4 \\
3,5\end{array}$ & $\begin{array}{l}8,7 \\
2,7\end{array}$ & $\begin{array}{l}8,2 \\
1,7\end{array}$ & $\begin{array}{l}8,2 \\
3,6\end{array}$ \\
\hline
\end{tabular}

Fonte: EPM/MS - Estudo Multicêntrico da prevalência do diabetes mellitus no Brasil.

te referidas, constatou-se maior presença de diabetes na área mais pobre, Santo Amaro. Embora esta diferença não fosse estatisticamente significante para o conjunto da amostra, ressalta-se o comportamento significantemente diferenciado da prevalência do DMAR na população masculina e feminina ( $p \varangle 0,01$ e $p<0,05$, respectivamente). Na população masculina, o maior valor foi observado na área de melhores condições de existência, Vila Mariana, enquanto que, na população feminina, os maiores valores foram observados na área intermediária (Saúde), seguida da área de mais baixas condições de existência (Santo Amaro).

Quanto à história familiar de diabetes, observou-se maior prevalência de DMAR entre aqueles que referiram sua presença, tanto para o sexo masculino como para o sexo feminino, sendo as diferenças significantes $(p<0,001)$. Estes resultados não se alteraram quando os dados foram ajustados por idade.

Vale dizer que, enquanto a variável história familiar de diabetes não apresentou relação sistemática com a idade, chamou a atenção sua associação com a escolaridade e a cor. No caso da escolaridade, $39,8 \%$ dos indivíduos com escolaridade acima de oito anos de estudo referiram a presença de história de diabetes na família, enquanto que esta proporção foi de $22,6 \%$ nos níveis abaixo de oito anos de estudo. Na população masculina, as proporções de referências à história familiar de diabetes, nos níveis acima e abaixo de oito anos de estudo, foram de $34,4 \%$ e $22,1 \%$, respectivamente, e de $44,8 \%$ e $23 \%$ na população feminina. Com relação à cor, maior presença de história familiar foi referida por brancos $(32,5 \%)$ do que por negros $(17,0 \%)$. Na população masculina, estes valores foram de $29,7 \%$ e $17,2 \%$, respectivamente, e, na população feminina, de $34,6 \%$ e $16,9 \%$. Todas estas diferenças foram estatisticamente significantes ( $p \varangle 0,001)$.

No tocante à análise multivariada, procedeu-se à dicotomização das variáveis. No caso da idade, considerou-se o grupo maior e menor de 50 anos; no caso da escolaridade, levaram-se em conta as categorias com menos e com mais de oito anos de estudo; com relação 
à cor, os dados foram reclassificados em brancos e não-brancos; e, em relação às áreas, procedeu-se à consideração de Santo Amaro em contraposição às demais àreas ( Vila Mariana e Saúde).

O processo adotado para hierarquização das variáveis revel ou a seguinte ordem de entrada na composição do modelo: idade, história familiar de diabetes, sexo, área e cor. Não chegaram a integrar o modelo as variáveis ocupação, naturalidade e escolaridade.

Dentre as cinco variáveis mais importantes, somente as três primeiras - idade, história familiar e sexo - foram consideradas, para efeito da análise multivariada, dada a rarefação dos dados, evidenciando o peso relativamente menor das demais variáveis sociais.

De acordo com os dados da Tabela 4, na qual se encontram distribuídas as pessoas com e sem DMAR, nas diferentes combinações, calculou-se o odds-ratio. A análise do peso das associações nestas situações permitiu constatar a presença sistemática de maior prevalência de DMAR nos grupos com história familiar da doença, independentemente de idade e sexo. Por outro lado, destacou-se a presença de maior prevalência do DMAR entre as mulheres, excetuando-se a situação do grupo de mais de cinqüenta anos de idade e com história familiar de diabetes.

\section{Considerações finais}

A importância da idade na configuração do modelo multivariado de análise, confirmando tendência observada em outras investigações (WHO, 1985; Everhart et al., 1985; Harris et al, 1987), não pode deixar de envolver a consideração, ao lado dos mecanismos biológicos, da associação da presença de idosos com a não-ocupação e com níveis mais baixos de escolaridade, o que reflete diferenciais intergeracionais de oportunidades de acesso ao ensino em nosso meio (Goldenberg, 1991). Acrescente-se a estas considerações, o dimensionamento da crescente demanda por serviços médicos, em função do envelhecimento populacional em curso no país (Ramos, 1993).

A história familiar de diabetes foi a segunda variável a ser incluída no modelo, valendo, a propósito, duas ordens de observações. A primeira refere-se ao fato de os diabéticos estarem, provavelmente, mais sensibilizados para a identificação de familiares com a doença do que os não-diabéticos. A segunda diz respeito à relação desta variável com a escolaridade e com a cor, sugerindo que, além de indicador genético da doença, no plano sócio-econômico, esta variável também seria indicativa das condições de acesso ao diagnóstico, independentemente da idade e do sexo, particularmente num país marcado por intensas desigualdades sociais.

No tocante ao sexo, terceira variável que integrou o modelo, destacou-se a maior prevalência do DMAR na população feminina. Este achado, aparentemente, contraria o resultado geral da prevalência detectado no estudo multicêntrico para o conjunto da população brasileira amostrada, porém deve-se levar em conta a especificidade da consideração da doença auto-referida ou pré-diagnosticada. Em que pese às indicações sobre a diversidade dos resultados existentes, vários autores (Everhart et al., 1985; Hamman, 1983) atribuem as situações de

Freqüência de DMAR, razão de probabilidades e odds-ratio. EMPDMB/ Fase 1 - Amostra São Paulo - $1986 / 87$.

\begin{tabular}{|c|c|c|c|c|c|c|}
\hline \multirow[t]{2}{*}{ Idade } & \multirow[t]{2}{*}{ Histórico Familiar } & \multirow[t]{2}{*}{ Sexo } & \multicolumn{2}{|c|}{ Diabetes } & \multirow[t]{2}{*}{ Razão de Probabilidade } & \multirow[t]{2}{*}{ Odds Ratio } \\
\hline & & & Sim & Não & & \\
\hline \multirow[t]{4}{*}{$30-4$} & Não & Masc. & 1 & 393 & 0,0025 & 1,00 \\
\hline & & Fem. & 9 & 524 & 0,0172 & 6,88 \\
\hline & Sim & Masc. & 6 & 168 & 0,0357 & 14,28 \\
\hline & & Fem. & 14 & 217 & 0,0645 & 25,80 \\
\hline \multirow[t]{4}{*}{$50 \geq$} & Não & Masc. & 10 & 178 & 0,0562 & 22,48 \\
\hline & & Fem. & 1 & 223 & 0,0942 & 37,67 \\
\hline & Sim & Masc. & 11 & 56 & 0,1964 & 78,56 \\
\hline & & Fem. & 22 & 149 & 0,1477 & 59,08 \\
\hline
\end{tabular}

Fonte: Estudo Multicêntrico sobre a prevalência do diabetes mellitus no Brasil. 
maior prevalência de DM AR entre as mulheres à maior freqüência das mesmas aos serviços de saúde. Estas considerações são sugestivas, diante das proporções mais elevadas de DMAR observadas na população feminina da amostra, que corresponderam a 58\% da prevalência global, enquanto que, entre os homens, esta proporção foi da ordem de $42 \%$.

Neste sentido, foram elucidativos os dados colhidos na subamostra da segunda fase do inquérito domiciliar realizado em São Paulo, envolvendo os diabéticos auto-referidos, mais os indi víduos que apresentaram glicemia de jejum $\geq 100$ mg no rastreamento, e $1 / 6$ daqueles que apresentaram glicemia $<100 \mathrm{mg}$. Levandose em conta a última consulta médica referida pelos entrevistados, os resultados revelaram que as mulheres freqüentavam mais os serviços de saúde do que os homens no intervalo dos últimos doze meses. Esta proporção foi de $90,4 \%$ entre as mulheres e de $74,6 \%$ entre os homens, sendo esta diferença estatisticamente significante $(p<0,01)$. Vale dizer, por outro lado, que as diferenças desapareceram quando da consideração específica da população de diabéticos já diagnosticados, permanecendo, neste caso, as proporções de freqüência à última consulta, no intervalo inferior a um ano, em torno de $96,5 \%$, tanto entre homens, como entre mulheres.

Quanto aos motivos das consultas, independentemente da data de sua realização, as mulheres dividiram suas respostas eqüitativamente entre as alternativas: "exames periódicos" e "problemas de saúde". Na população masculina, porém, 76,7\% fizeram referência aos "problemas de saúde" como motivo de mobilização para a busca de assistência médica, ressaltando-se que esta diferença também desapareceu quando foram considerados, exclusivamente, os diabéticos.

A maior freqüência das mulheres aos serviços de saúde, inclusive nas condições mais precárias de existência, não só justificaria a concentração do DMAR nos estratos de baixos níveis sócio-econômicos, como poderia explicar os valores mai ores da prevalência do DMAR na população feminina em relaçào à masculina. De forma correlata, levando-se em conta o maior desconhecimento, ou falta de diagnóstico, entre os homens, seria de se esperar que os diferenciais de prevalência, segundo gênero, desaparecessem, na medida em que fossem considerados não só o diabetes conhecido, como o desconhecido, o que deverá constituir objeto de estudo a ser explorado proximamente.

Resultados relativos às áreas, quarta variável na ordem de importância, ainda que não ti- vessem integrado o modelo, dada a rarefação dos dados, apontaram para a distribuição diferenciada do DMAR nas populações masculina e feminina. No caso da população masculina, a concentração na área de nível sócio-econômico mais elevado seria coerente com a associação do DMAR à presença de história familiar de diabetes, a qual estaria associada, por sua vez, aos melhores níveis de escolaridade. Entre as mulheres, esta associação estaria relativizada diante do hábito de procurar serviços médicos com maior freqüência do que os homens. Conforme apontam estudos antropológicos em nosso meio (Fausto Neto, 1982; Woortmann, 1983), constitui um atributo feminino da divisão sexual do trabalho providenciar assistência médica para os integrantes da família, o que garantiria seu acesso aos serviços médicos nas situações mais precárias de existência.

Dados relativos à cor, quinta variável em ordem de importância a ser incluída no modeIo, mas igualmente descartados em função da rarefação dos dados, apontaram para a mesma direção dos resultados observados anteriormente. Considerando a associação da cor com a escolaridade e com a distribuição por área, a maior prevalência da doença na população masculina branca e na população feminina não-branca também seria indicativa do diferencial de prevalência do DMAR entre homens e mulheres, atrelada a distintas situações sócio-econômicas: nos níveis mais altos, entre os homens, e, nos mais baixos, entre as mulheres.

Estas indicações não permitiriam descartar a possibilidade da existência de uma distribuição desigual da prevalência do DMAR nos distintos estratos sociais. A considerar tais observações, poder-se-ia aventar a hipótese de que, ao incorporar na consideração da prevalência não só os diabéticos pré-diagnosticados, como também os recém-diagnosticados, a presença do diabetes deveria se ampliar nos estratos de menor nível de renda. Esta hipótese se aplicaria, particularmente, à população masculina, cujo desconhecimento da doença foi maior do que entre as mulheres, tendo como pressuposto que o acesso ao diagnóstico, entre outras facetas dos diferenciais sociais de existência, teria um papel indiscutível nas estimativas do diabetes mellitus em nosso meio. Isto equivaleria dizer que, mediante a implementação de formas diagnósticas mais ativas nos serviços existentes, a democratização da assistência permitiria dimensionar uma maior prevalência nos estratos menos favorecidos do que as observadas no momento, corroborando os indícios apontados na análise do DMAR, 
segundo as variáveis sociais consideradas isoladamente.

Dentre as variáveis que nem foram apontadas para ingressar no modelo, a situação ocupacional, dada a importância evidenciada na análise individualizada, merece algumas observações. Tendo em vista que o não-trabalho se associa, em geral, à perda da função social produtiva em torno da qual se organizam as funções vitais, físicas e mentais (Garcia, 1983), a associação da não-ocupação com a maior presença de DMAR guarda coerência com a epidemiologia da doença. Neste sentido, cabe lembrar que a maior freqüência de DMAR entre os não-ocupados (geralmente aposentados), na população masculina, também se associa à idade, o que acaba por confundir a participação da referida variável na consideração da manifestação da doença.

Na população feminina, por outro lado, elevada proporção de mulheres estaria inserida na categoria de não-ocupadas, exercendo atividades domésticas, o que evidenciaria o sentido distinto de sua consideração neste contingente populacional, ressaltando-se, inclusive, a estrutura etária mais jovem das mesmas do que a dos homens, nesta mesma categoria. Re-

\section{Referências}

BUSSAB, W. O. \& DINI, N. P., 1985. Pesquisa de emprego e desemprego. SEADE/DIESE: Regiões homogêneas da Grande São Paulo. Revista da Fundação SEADE, 1:5-11.

EVERHART, J., KNOWLER, W. C., BENNETT, P. H. \& F. R. C. P., 1985. Incidence and risk factors for noninsulin-dependent diabetes. In: National Diabetes Data Group. Diabetes in America. (NIH Publication) pp. 85-1468. Washington D.C.: U.S. Department of Health and Human Service, National Institute of Health.

FAUSTO NETO, A. M. Q., 1982. Família Operária e Reprodução da Força deTrabalho. Petrópolis: Editora Vozes.

FIBGE (Fundação Instituto Brasileiro de Geografia e Estatística), 1983. Censo Demográfico de 1980. Rio de Janeiro: FIBGE.

FIBGE (Fundação Instituto Brasileiro de Geografia e Estatística), 1988. Pesquisa de Orçamentos Familiares, 1986/87. Rio de Janeiro: FIBGE.

FIBGE (Fundação Instituto Brasileiro de Geografia e Estatística), 1990. Pesquisa Nacional por Amostra de Domicílios 1987. Rio de Janeiro: FIBGE.

GARCIA, J. C., 1983. La categoria trabajo en la medicina. Cuadernos Médico-Sociales: 23:5-17.

GOLDENBERG, P., 1991. Condiciones de vida y salud en los años 80. Reproducción como categoria de análisis. Cuadernos Médico-Sociales, 5:35-54. lativizar o significado da não-ocupação, na consideração da prevalência do DMAR na população feminina, não implica descartar sua importância, haja vista a maior prevalência do DMAR na área da Saúde, que concentrou a maior proporção de donas-de-casa. Também vale dizer que as mulheres negras, as quais apresentam maiores níveis de ocupação e menores níveis de escolaridade do que as brancas, em que pese serem mais jovens, a maior prevalência entre elas levanta a suspeita de que a inserção no mercado de trabalho pode se constituir numa sobrecarga, em condições sócio-econômicas desfavoráveis e em meio aos demais encargos domésticos, que, tradicionalmente, recaem sobre as mulheres.

Esta face da assimetria de gênero aponta para a especificidade da questão do trabalho feminino, em nossa sociedade, evidenciando distintos significados, atribuíveis à mesma categoria em ambos os gêneros, na forma como foi tratada no presente estudo. Nestas condições, ela concorre com um caráter que causa confusão na aferição de seus efeitos na estimativa da prevalência do diabetes, só identificável mediante estudos específicos.

HAMMAN, R. F., 1983. Diabetes in afluent societies. In: Diabetes in Epidemiological Perspectives (Mann, J. I.; Pyorala, K. \& Teuscher, A., eds.), pp. 7-42. Edinburgh/London: Churchill Livingstone. HARRIS, M. I., HADDEN, W. C. \& BENNET, P. H., 1987. Prevalence of diabetes and impaired glucose tolerance and plasma glucose level in population aged 20-74 yr. Diabetes, 36:523-534.

HIGGINS, J. E. \& KOCH, G. G., 1975. Variable selection and generalized chi-square analysis of categoriacal: data as used to model byssinosis prevalence. International Statistical Review, 45:51-62.

MALERBI, D. A. \& FRANCO, L. J., 1992. Multicenter study of the prevalence of diabetes mellitus and impaired glucose tolerance in the urban Brazilian population aged 30-69 yr. Diabetes Care, 15:15091516.

RAM OS, L. R., 1993. A explosão demográfica da terceira idade no Brasil: uma questão de saúde pública. Gerontologia, 1:3-8.

WOORTM ANN, T., 1983. A Família Trabalhadora. VII Encontro Anual da Associação Nacional de PósGraduação e Pesquisa em Ciências Sociais. (mimeo).

WHO (World Health Organization), 1985.Diabetes Mellitus. Report of a WHO Study Group. Technical Report Series, no 727, Geneve: WHO. 